\title{
Zebrafish Bioassay-guided Microfractionation for the Rapid in vivo Identification of Pharmacologically Active Natural Products
}

\author{
Soura Challalsa, Nadine Bohni ${ }^{\mathrm{a}}$, Olivia E. Buenafe ${ }^{\text {bc }}$, Camila V. Esguerra ${ }^{\mathrm{b}}$, Peter A. M. de Witte ${ }^{\mathrm{b}}$, \\ Jean-Luc Wolfendera ${ }^{\mathrm{a}}$, and Alexander D. Crawford ${ }^{\mathrm{b}}$ \\ $\S S C S-D S M$ Award for best poster presentation
}

\begin{abstract}
The rapid acquisition of structural and bioactivity information on natural products (NPs) at the submilligram scale is key for performing efficient bioactivity-guided isolations. Zebrafish offer the possibility of rapid in vivo bioactivity analysis of small molecules at the microgram scale - an attractive feature when combined with high-resolution fractionation technologies and analytical methods such as UHPLC-TOF-MS and microflow NMR. Numerous biomedically relevant assays are now available in zebrafish, encompassing most indication areas. Zebrafish also provide the possibility to screen bioactive compounds for potential hepato-, cardio-, and neurotoxicities at a very early stage in the drug discovery process. Here we describe two strategies using zebrafish bioassays for the high-resolution in vivo bioactivity profiling of medicinal plants, using either a one-step or a twostep procedure for active compound isolation directly into 96 -well plates. The analysis of the microfractions by microflow NMR in combination with UHPLC-TOF-MS of the extract enables the rapid dereplication of compounds and an estimation of their microgram quantities for zebrafish bioassays. Both the one-step and the two-step isolation procedures enable a rapid estimation of the bioactive potential of NPs directly from crude extracts. In summary, we present an in vivo, microgram-scale NP discovery platform combining zebrafish bioassays with microscale analytics to identify, isolate and evaluate pharmacologically active NPs.
\end{abstract}

Keywords: Microflow NMR · Microfractionation · Natural products · UHPLC-TOF-MS · Zebrafish

\section{Introduction}

Natural products (NP) are an important resource for drug discovery and development - as therapeutic agents, as drug leads, and as pharmacological tools for target discovery and validation. The chemical diversity of NPs has contributed significantly to

\footnotetext{
${ }^{*}$ Correspondence: Prof. J.-L. Wolfendera, Dr. A. Crawford ${ }^{b}$

aphytochimie et Produits Naturels Bioactifs

Ecole de Pharmacie Genève-Lausanne

Section des Sciences Pharmaceutiques

Université de Genève

Quai Ernest-Ansermet 30

$\mathrm{CH}-1211$ Genève 4

Tel.: +4122 3793385

Fax: +412237933 99

E-mail: jean-luc.wolfender@unige.ch

bLaboratory for Molecular Biodiscovery

Department of Pharmaceutical \& Pharmacologica

Sciences

University of Leuven

Herestraat 49, P.O. Box 824

BE-3000 Leuven, Belgium

Tel.: +32 16323439

Fax: +3216323460

E-mail: alexander.crawford@pharm.kuleuven.be

'Department of Chemistry

Ateneo de Manila University, Manila, Philippines
}

the development of drugs for a wide range of diseases. ${ }^{[1]}$

NP research and drug discovery often begins with the source itself - plants, fungi, and microbes, as well as marine flora and fauna. The investigation can be guided e.g. by a plant's ethnopharmacological use, or by a molecule category known to provide a given activity (chemotaxonomic targeting). Numerous efforts to optimize screening paradigms are enabling more efficient drug discovery based on NPs - for example, the use of small-molecule libraries, ${ }^{[2]}$ to decrease the time for isolation and purification; and the improvement of analytical methods for structure elucidation and dereplication, ${ }^{[3]}$ thereby decreasing sample amounts needed for analysis. ${ }^{[4]}$ Bioactivity-guided fractionation facilitates the isolation and identification of active compounds as well as the assessment of the compound toxicities, which is a crucial factor for further investigations. ${ }^{[5]}$

Analyzing an NP matrix is a challenging task in many respects. Particularly plant extracts are complex mixtures which can contain several closely related compounds eluting in a narrow chromatographic area in metabolite profiling analysis. In addi- tion, main constituents can mask minor compounds responsible for the targeted bioactivity.

Classical bioactivity-guided fractionation has provided a good means to solve such issues. However, such an approach requires multiple chromatographic steps and large amounts of biological material. ${ }^{[5]}$ This process is therefore labor-intensive and is generally regarded to be too slow to cope with most high-throughput screening paradigms..$^{[4]}$

In an effort to partially solve some of these issues, we report here the novel combination of microfractionation procedures with an in vivo bioassay platform to enable the rapid generation of both structural and bioactivity information from limited amounts of biological material.

\section{Zebrafish as an in vivo Model for Microgram-scale NP Discovery}

In contrast to enzymatic or cell-based reporter assays, high-content bioassays (e.g. phenotypic assays using cells or organisms) allow the hypothesis-independent analysis of pharmacological activity. 
Particularly in vivo models offer the possibility to screen for biomedically relevant bioactivities in a target- and pathway-independent manner. Nevertheless, mammalian models such as rodents require larger amounts of compound (in the milligram range) for activity analysis, and are therefore not ideal in vivo platforms for bioassay-guided fractionation.

The recent emergence of in vivo, microgram-scale, high-throughput assays based on zebrafish embryos and larvae represents a unique opportunity for the rapid identification of bioactive NPs. Embryos and larvae of the zebrafish (Danio rerio, a tropical freshwater teleost) have within the past two decades emerged as an ideal model system not just for developmental and biomedical genetics, but also for chemical genetics and drug discovery. ${ }^{[6]}$ Although zebrafish were first proposed as a model for small-molecule discovery in $1957,{ }^{77]}$ it has only been in the last ten years that compound screens carried out using zebrafish assays have identified a growing number of bioactive small molecules in a variety of indication areas. ${ }^{6,8]}$ More recently, we and others have established zebrafish as an in vivo platform for NP discovery, ${ }^{[9,10]}$ both for bioassay-guided fractionation as well as for screening prefractionated NP libraries.
Zebrafish offer several advantages as model organisms - most importantly, they are a well-characterized vertebrate species with a fully-sequenced genome, and produce large numbers of rapidly and synchronously developing transparent embryos and larvae. The rapid development of zebrafish ensures that all biomedically relevant tissues and organs form and achieve functionality within the first few days after fertilization. Of equal importance, zebrafish embryos and larvae are small (1-4 mm, depending on the developmental stage) and therefore compatible with 96-well and even 384-well plates, thereby reducing the amount of compound required for this in vivo assay to the level of micrograms (depending on the potency of the compound, as little as 1 microgram may be sufficient for an initial bioactivity screen in zebrafish, approximately 1000-fold less than what is required for a typical dose in mice). This latter feature is key for NP discovery, as many high-resolution separation methods based on HPLC, particularly microfractionation, result in very limited sample sizes that would otherwise be insufficient for the in vivo analysis of bioactivity.

Bioassays based on zebrafish embryos and larvae are now available for a wide range of indication areas (see recent reviews ${ }^{[6,9]}$. Briefly, zebrafish models have been developed for the analysis of inflammation, cancer, angiogenesis, diabetes, epilepsy, and several other diseases. ${ }^{[11,12]}$ Many of these models are potentially useful as in vivo assays for high-throughput screens of small molecules including NPs. Additionally, recent developments include zebrafish assays in the areas of bacterial and viral infection, alcoholism, and behavioral analysis (Fig. 1).

The ability to carry out in vivo assays in zebrafish at the microgram scale presents a unique opportunity to take advantage of analytical and microfractionation methods that have recently been developed at this level as well. The effective combination of these platforms will enable the rapid identification of NPs based on their pharmacological activity in vivo - an attractive possibility to expand the current scope of NP discovery.

\section{Hyphenated Chromatographic Methods for Crude Extract Profiling and Microfractionation}

\section{UHPLC-TOF-MS for Metabolite Profiling}

Crude plant extracts are complex mixtures containing a high number of NPs of various biosynthetic origins. In order to

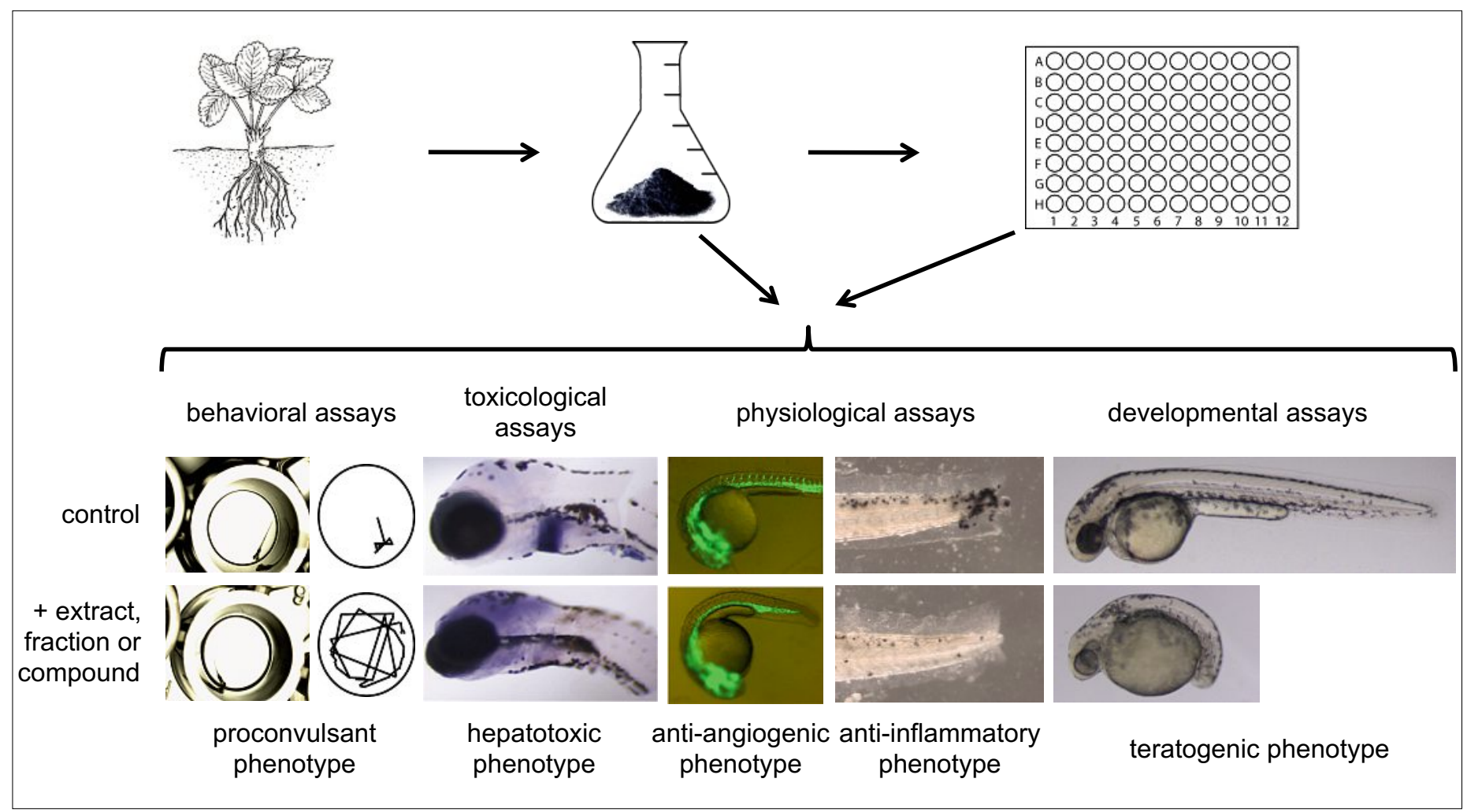

Fig. 1. Zebrafish-based screening of crude extracts, fractions, and pure NPs. A wide variety of zebrafish assays are now available, including behavioral, toxicological, physiological and developmental assays. Most of these bioassays are suitable for NP discovery at the microgram scale, and can be used to screen crude extracts, chromatographic fractions (including microfractions), and pure compounds. Depicted above are zebrafish assays for epilepsy (automated video tracking of locomotor activity and seizure behavior), hepatotoxicity (whole-mount in situ hybridization analysis of the liver-specific fatty acid binding protein fabp10a), angiogenesis (visualization of intersegmental vessel outgrowth in a fli-1:EGFP transgenic line with vasculature-specific fluorescence), inflammation (visualization of leukocyte migration after tail transection via myeloperoxidase staining), and developmental signaling pathways (morphological analysis). 
rapidly estimate the secondary metabolite composition of these extracts, methods providing high chromatographic resolution and online spectroscopic information are required. In this respect, hyphenated techniques such as Ultra-High-Performance Liquid Chromatography (UHPLC) coupled with high-resolution Time-Of-Flight Mass Spectrometry (TOF-MS), represent a state-of-the-art analytical platform both for profiling/dereplication studies as well as metabolomics. [13] UHPLC uses columns packed with sub $2 \mu \mathrm{m}$ particles and high operating pressures (up to 1000 bar) compared to standard HPLC. This results in a decrease in analysis time (e.g. for highthroughput analysis of many microfractions) or an increase in peak capacity (for in-depth profiling studies).

TOF-MS detection on the other hand provides a sensitive and universal detection of most NPs and its high mass-accuracy and high resolution gives the possibility to assign molecular formula on-line, a key element for dereplication. ${ }^{[3]}$

The combination of high-resolution UHPLC with TOF-MS is an ideal tool for profiling complex biological and microbial extracts, and all spectroscopic information is obtained with microgram amounts of crude extract while the detection of NPs is realized in the nano- or picogram range.

\section{Semi-preparative LC-MS for Microfractionation}

In order to profile activity in a crude plant extract, NPs have to be obtained and isolated in the microgram range. This range is compatible both with in vivo assays in small organisms such as those presented with zebrafish, as well as de novo structure identification and absolute quantification that can be performed with state-of-the-art microflow NMR methods (see below).

In order to rapidly obtain microfractions of such amounts, semi-preparative HPLC is well suited since it provides higher loading capacities than analytical HPLC and reasonably high-resolution separation. Chromatographic gradients can be transferred from the UHPLC analytical level to the semi-preparative level to obtain similar separation selectivity. Semipreparative HPLC is thus a suitable method for an efficient rapid microfractionation of crude plant extract with no or reduced sample preparation. ${ }^{[14]}$ Semi-preparative HPLC combined to MS detection enables the separation of a wide variety of compound classes to be monitored and to correlate the information with that from the UHPLC-TOF-MS profiling for an efficient localization of the compounds of interest.

\section{Microfractionation Strategies}

With the aim of rapidly localizing and identifying bioactive compounds either a one-step or a two-step microfractionation strategy can be applied according to the complexity and the nature of a given crude extract. Several plant extracts have been studied in this way to identify either antiangiogenic NPs based on a physiological zebrafish assay or anti-convulsant NPs based on a behavioral zebrafish assay.

For example, in an ongoing search for anti-angiogenic compounds from a Tanzanian medicinal plant (Fabacea), we employed a one-step microfractionation strategy. Dereplication by UHPLC-TOFMS revealed the presence of isoflavones and other metabolites widespread through-
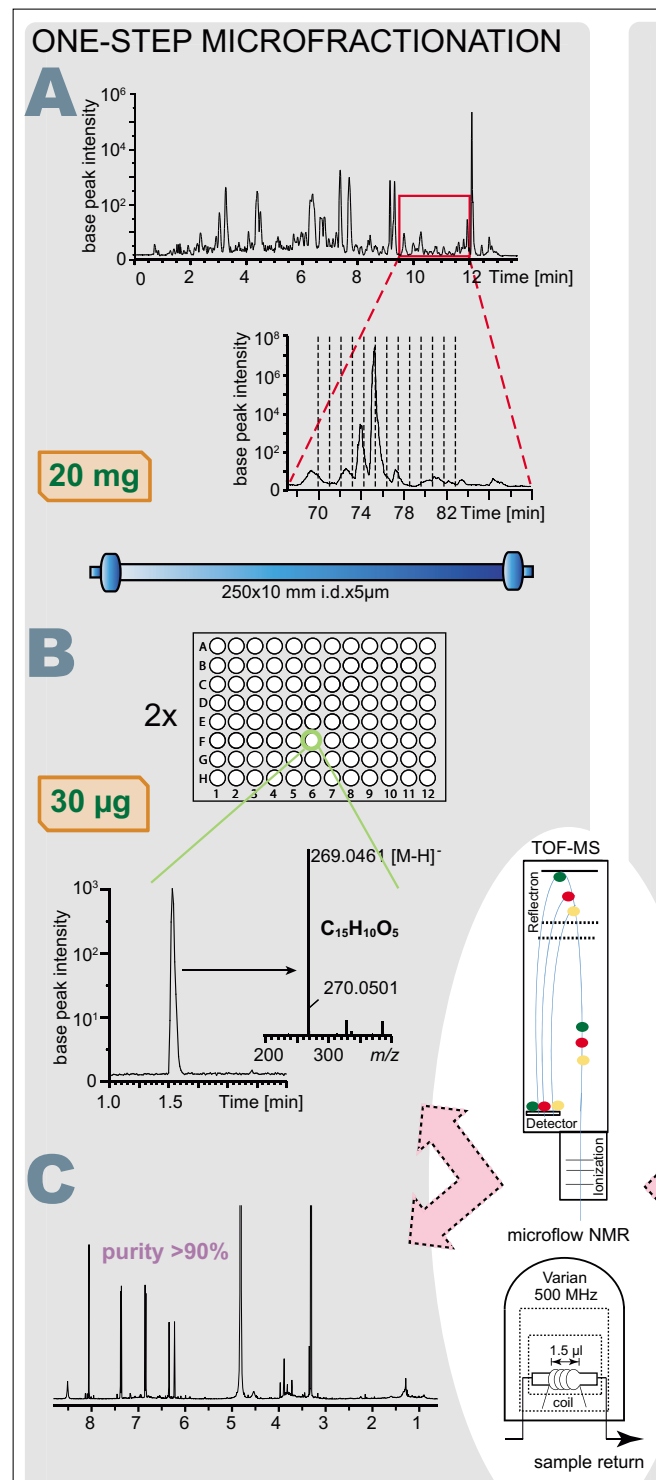

TWO-STEP MICROFRACTIONATION preparative HPLC-MS $(100 \times 2.1 \mathrm{~mm}$ to $250 \times 10 \mathrm{~mm} \mathrm{C18}$ column) enabled the isolation of bioactive compounds from 20 mg of crude extract in one step. The collection was done directly into two 96-deep well plates. Aliquots were taken for testing the microfractions for inhibition of angiogenesis in zebrafish and for subsequent high-throughput UHPLC-TOF-MS analysis of the microfractions of interest (Fig. 2B). The major part of the microfractions was kept for microflow NMR analysis (Fig. 2C). Several microfractions showed
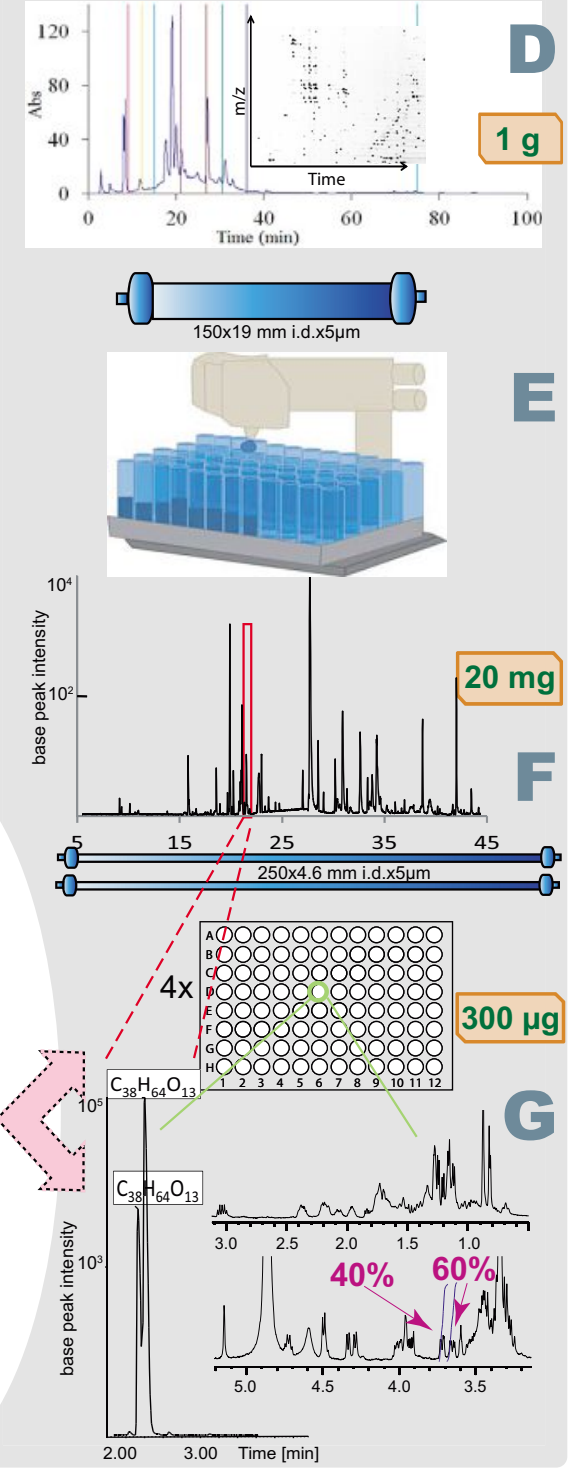

Fig. 2. A: UHPLC-TOF-MS profiling of $1 \mu \mathrm{g}$ of the crude extract of a Fabaceae species and generation of microfractions using semi-preparative HPLC monitored by MS. B: Collection of microfractions into 96-deep well plates and characterization of compounds of interest by UHPLC-TOF-MS. C: Structure elucidation and quantification by microflow NMR. D: UHPLC-TOF-MS profiling of $1 \mu \mathrm{g}$ of the crude extract of a Solanacea species for the gradient transfer onto the semi-preparative HPLC monitored by UV. E: Collection of fractions into tubes. F: Microfractionation of the active fraction by HPLC-MS using two analytical columns coupled in series. Separation optimized by calculation based on two generic UHPLC gradients of different slopes. G: Structure elucidation and quantification of the active microfraction containing two isomeric compound by UHPLCTOF-MS and microflow NMR. 
an inhibition of angiogenesis in zebrafish and contained only single compounds all bearing an isoflavone skeleton. ${ }^{[15]}$

A different strategy has been used for the identification and isolation of anticonvulsant compounds from a Philippine medicinal plant (Solanaceae). Compared to the Fabaceae extract discussed above, the UHPLC-TOF-MS profile was more complex and showed the presence of related isomers partially coeulting (Fig. 2D). Since in this case a high LC resolution was needed, the gradient elution was optimized by performing two generic gradients of a slope of 1 and $3 \%$ and these data were used to create an optimal gradient by software calculation. ${ }^{[16]}$ A first enrichment was performed on a semi-preparative HPLC column of $150 \times 19 \mathrm{~mm}$ generating nine fractions (Fig. 2E). The active fraction on the zebrafish anti-convulsant assay corresponded to a zone with no UV active peaks. The MS data of this region revealed the presence of different high molecular weight compounds (see inset 2D ion map plot, Fig. 2D). Based on the optimized gradient calculated from the two profiling gradients, a high-resolution separation of the active fraction was performed on two $250 \times 4.6 \mathrm{~mm}$ columns coupled in series $(500 \times 4.6 \mathrm{~mm})$ (Fig. $2 \mathrm{~F})$. As shown in Fig. 2F, a high-resolution profiling was obtained on $20 \mathrm{mg}$ of the active fraction. All compounds eluting in this second separation were collected into four 96-deep well plates and the activity was concentrated in well-defined microfractions containing some of the high molecular weight compounds. Even with this very high resolution separation the active fraction was found to contain two isomeric structures related to steroid glycosides..$^{[17]}$

With both microfractionation strategies, microflow NMR analysis was used to confirm the identity of the compound isolated and to quantify the amount present in each fraction at the microgram level (Fig. 2G).

\section{Microflow NMR for Identification and Quantification}

NMR spectroscopy is the method of choice for the identification and structure elucidation of most small molecules. In combination with high-resolution MS, an unambiguous assignment is often possible. But while mass spectrometric methods are highly sensitive and require only nanogram amounts of sample, NMR is an insensitive technique. However, considerable efforts have been made in the last twenty years to improve its sensitivity either by reducing the noise level generated by the probes (cryo-probes) or by optimizing the cell design for maximizing filling factor and diameter (microflow NMR probes).

Thus, these recent probe technologies in combination with high field magnets enable the de novo structure isolation of NPs with a few micrograms of sample. ${ }^{[18]}$

In the strategy presented, a microflow probe (CapNMR ${ }^{\mathrm{TM}}$ ) was used for structure elucidation of the compounds isolated in the bioactive microfractions. Here, all dried microfractions were solubilized in less than $10 \mu \mathrm{l}$ of deuterated solvent and analyzed automatically by using an automated sample injection unit (One Minute-

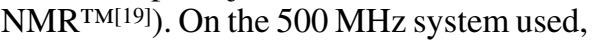
this allows for $2 \mathrm{D}$ experiments and de novo structure elucidation on as little as 50-100 $\mu \mathrm{g}$ of sample.

Assessment of the bioactive potency of unknown NPs obtained by microfractionation is a challenging task, since their quantities have to be correctly estimated. Weighing the microfractions is not only impractical, but also inaccurate at submilligram quantities. Using NMR, sample identification and quantification ${ }^{[20]}$ can be combined in one analysis step. NMR quantification can be performed either with an internal standard, using the ERETIC method, or the PULCON ${ }^{[21]}$ method with reference to an external standard. PULCON correlates the absolute intensities of two spectra measured in different solution conditions and was used for the estimation of the amount of bioactive NPs collected.

By applying this method, the bioactivity measured could be accurately related to the microgram amount tested and the purity of the microfractions could be assessed (Fig. 2C) . In the case of coeluting NPs found in the same microfraction (Fig. $2 \mathrm{~F}$ ), each compound was quantified and its relative amount was estimated.

\section{NP Discovery Platform Combining in vivo Zebrafish Assays with Microscale Analytics}

We have described herein the development of an integrated platform for the rapid acquisition of structural and in vivo bioactivity information on sub-milligram amounts of NPs from crude extracts. The combination of sensitive and rapid analytical techniques (UHPLC-TOF-MS and microflow NMR) with bioassays in small organisms (especially zebrafish) provides a powerful strategy for the rapid identification and quantification of NPs and the rapid analysis of their pharmacological activity in vivo. Zebrafish are an attractive in vivo assay for NP discovery because of their small size and their strong similarities with humans from a genetic, physiological and pharmacological point of view. These features enable the use of only microgram amounts of active compounds, fractions, and crude extracts for bioactivity analysis using a large and growing panel of biomed- ically relevant in vivo assays (morphological, physiological, and behavioral). Such strategies may improve drug discovery efforts based on NPs by enabling the more efficient identification of novel, bioactive lead molecules.

Received: January 20, 2012

[1] A. L. Harvey, Drug Discov. Today 2008, 13, 894.

[2] G. R. Eldridge, H. C. Vervoort, C. M. Lee, P. A. Cremin, C. T. Williams, S. M. Hart, M. G. Goering, M. O’Neil-Johnson, L. Zeng, Anal. Chem. 2002, 74, 3963.

[3] J.-L. Wolfender, G. Marti, E. Ferreira Queiroz, Curr. Org. Chem. 2010, 14, 1808.

[4] F. E. Koehn, G. T. Carter, Nat. Rev. Drug Discovery 2005, 4, 206.

[5] K. Hostettmann, A. Marston, Chimia 2007, 61, 322.

[6] L. I. Zon, R. T. Peterson, Nat. Rev. Drug Discovery 2005, 4, 35.

[7] R. W. Jones, M. N. Huffman, Trans. Am. Microsc. Soc. 1957, 76, 177.

[8] R. T. Peterson, B. A. Link, J. E. Dowling, S. L. Schreiber, PNAS 2000, 97, 12965.

[9] A. D. Crawford, C. V. Esguerra, P. A. M. de Witte, Planta Med. 2008, 74, 624.

[10] A. D. Crawford, S. Liekens, A. R. Kamuhabwa, J. Maes, S. Munck, R. Busson, J. Rozenski, C. V. Esguerra, P. A. M. de Witte, PLoS ONE 2011, 6, e14694.

[11] P. W. Ingham, Hum. Mol. Genet. 2009, 18, R107.

[12] G. J. Lieschke, P. D. Currie, Nat. Rev. Genet. 2007, 8, 353 .

[13] P. J. Eugster, D. Guillarme, S. Rudaz, J.-L. Veuthey, P.-A. Carrupt, J.-L. Wolfender, J. AOAC Int. 2011, 94, 51.

[14] J.-L. Wolfender, Planta Med. 2009, 75, 719.

[15] A. D. Crawford, N. Bohni, J. Maes, M. L. Cordero Maldonado, A. R. Kamuhabwa, M. J. Moshi, C. V. Esguerra, P. A. M. de Witte, J.-L. Wolfender, manuscript in preparation.

[16] G. Glauser, D. Guillarme, E. Grata, J. Boccard, A. Thiocone, P.-A. Carrupt, J.-L. Veuthey, S. Rudaz, J.-L. Wolfender, J. Chromatogr. A 2008, $1180,90$.

[17] A. D. Crawford, S. Challal, O. E. Buenafe, A. L. Harvey, C. V. Esguerra, P. A. M. de Witte, J.-L. Wolfender, Planta Med. 2011, 77, 1348.

[18] J. F. Hu, E. Garo, H. D. Yoo, P. A. Cremin, L. Zeng, M. G. Goering, M. O'Neil-Johnson, G. R. Eldridge, Phytochem. Anal. 2005, 16, 127.

[19] A. Jansma, T. Chuan, R. W. Albrecht, D. L. Olson, T. L. Peck, B. H. Geierstanger, Anal. Chem. 2005, 77, 6509.

[20] D. S. Dalisay, T. F. Molinski, J. Nat. Prod. 2009, 72, 739.

[21] G. Wider, L. Dreier, J. Am. Chem. Soc. 2006, $128,2571$. 